\title{
Statin use and the risk of ovarian and endometrial cancers: a meta-analysis
}

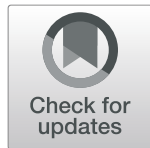

\author{
Yizi Wang, Fang Ren, Zixuan Song, Peng Chen, Shuang Liu and Ling Ouyang *i)
}

\begin{abstract}
Background: The relationship between statin use and the risk of ovarian or endometrial cancer remains controversial. Here, we investigated the relationship between statin use and the risk of ovarian and endometrial cancers.

Methods: We conducted a meta-analysis using articles retrieved from the PubMed, Embase, and Web of Science databases. All original comparative studies published in English that were related to statin use and the risk of ovarian or endometrial cancer were included.

Results: This meta-analysis included 19 studies enrolling 1,999,362 female subjects and 19,849 cancer cases (7,948 ovarian cancer cases and 11,901 endometrial cancer cases). The overall analysis indicated that statin use did not significantly reduce the risk of ovarian cancer [relative risk $(\mathrm{RR})=0.88,95 \%$ confidence interval $(\mathrm{Cl}) 0.76-1.03, p=0.12$ ] or the risk of endometrial cancer ( $R R=0.88,95 \% \mathrm{Cl} 0.78-1.00, p=0.05$.) Subgroup analyses based on study type, percentage of cancer cases, study location, and quality of studies also supported our conclusions. No association was found between long-term statin use ( $>5$ years) and the risk of ovarian cancer ( $\mathrm{RR}=0.73,95 \% \mathrm{Cl} 0.51-1.04, p=0.08)$ or endometrial cancer ( $\mathrm{RR}=0.79,95 \% \mathrm{Cl} 0.58-1.08, p=0.14)$.

Conclusions: Statin use did not lower the risk of ovarian cancer or endometrial cancer. The long-term use of statins ( $>5$ years) was not associated with a reduction in the risk of ovarian or endometrial cancer.
\end{abstract}

Keywords: Statin, Ovarian cancer, Endometrial cancer

\section{Background}

Ovarian and endometrial cancers are the two most commonly diagnosed gynecological cancers, with the highest mortality of all gynecological cancers [1]. Ovarian cancer is the leading cause of cancer death in gynecological malignancies and the fifth leading cause of all cancers in women in the US [2]. Endometrial cancer is the most common gynecological malignancy in the US, with a death rate that has increased gradually [3].

Esposito et al. reported in a meta-analysis that metabolic syndrome is associated with an increased risk of ovarian and endometrial cancers [4]. Hydroxymethylglutaryl-CoA reductase inhibitors (statins) are cholesterol-lowering drugs that are primarily used for hypercholesterolemia therapy. They also have antitumor effects, which have been reported in experimental research on many cancers [5]. In recent years, the pharmacological functions of

\footnotetext{
* Correspondence: ouyl@sj-hospital.org

Department of Obstetrics and Gynecology, Shengjing Hospital of China

Medical University, Shenyang 110004, People's Republic of China
}

statins were found to be effective in several female reproductive diseases [6-9]. Statins inhibit 3-hydroxy-3methylglutaryl coenzyme A (HMG CoA) reductase, the key enzyme in the mevalonate pathway for cholesterol synthesis. They can down regulate products of the mevalonate pathway, such as farnesyl diphosphate and geranyl diphosphate, which may contribute to regulating tumor cell growth, motility, and differentiation [10,11].

Epidemiological studies of statins and the risk of endometrial or ovarian cancers have shown inconsistent results. A recent population-based study of 161,808 postmenopausal women indicated that statin use can reduce the risk of endometrial cancer but not that of ovarian cancer [12]. In contrast, another study reported that statin use reduced the risk of ovarian cancer [13]. Previous studies have reported that statin use was associated with a reduced risk of ovarian or endometrial cancer [14, 15].

Here, we conducted a meta-analysis to investigate the relationship between statin use and the risk of endocrine-related gynecological cancers. 


\section{Methods}

\section{Search strategy}

This study was conducted in accordance with the Preferred Reporting Items for Systematic Reviews and Meta-Analyses (PRISMA) guidelines (http://www.prisma-statement.org/). A comprehensive search was performed of the PubMed, Embase, and Cochrane databases to August 1, 2018. The following $\mathrm{MeSH}$ and main keywords were used: "hydroxymethylglutaryl-CoA reductase inhibitor," "Reductase Inhibitors, Hydroxymethylglutaryl-CoA," "Inhibitors, HMG-CoA Reductase," "Reductase Inhibitors, HMG-CoA, " "HMG-CoA Reductase Inhibitors," "Statins, HMG-CoA," "Statins," "Hydroxymethylglutaryl-Coenzyme A Inhibitors," and associated terms; and "Ovarian Neoplasm," "Ovary Neoplasm," "Ovary Cancer," "Ovarian Cancer," "Cancer of Ovary," "Endometrial Neoplasm," "Endometrial Carcinoma, " "Endometrial Cancer," "Endometrium Cancer," "Endometrium Carcinoma," and associated terms. The language was restricted to English. For multiple-arm comparative studies, we extracted data only from the arms that matched our eligibility criteria. We also performed manual searches of the reference lists of the selected studies to retrieve all relevant data.

\section{Inclusion and exclusion criteria}

Studies were selected according to the PICOS (population, intervention, comparison, outcomes, and study design) guidelines if they met the following inclusion criteria: (1) population: the entire population of women recruited to a certain trial, and the endpoint is incidence of ovarian or endometrial cancer; any histologic types of cancer were included for ovarian and endometrial cancers, including ovarian borderline malignancies; (2) intervention: patients used statins; (3) comparison: patients used statin versus control (placebo or no statin); (4) outcomes: studies compared morbidity between two groups using odds ratio (OR), relative risk (RR), or hazard ratio (HR) estimates with 95\% confidence intervals (CIs), or provided sufficient data to compute morbidity; and (5) study design: studies were comparative [i.e., randomized control trials (RCTs) or observational studies].

Exclusion criteria were as follows: (1) population: mixed patients with other cancer types or patients with indeterminate cancer; (2) intervention: patients did not use statins; (3) comparison: patients used statin versus other drugs; (4) outcomes: studies lacked morbidity data; and (5) study design: single-arm study without a control group.

\section{Data extraction and quality assessment of included studies}

Two reviewers (YW and FR) reviewed and assessed each of the included studies. Data extraction was performed independently. The other authors (ZS, PC, and SL) reviewed the included studies as well for inclusion. The following information was extracted from the included studies: first author, publication year, country of study, study type, cancer site and cases, number of subjects, percentage of cancer cases, study period, cases of statin use and control groups, follow-up time, and multivariable adjusted RR estimates with corresponding 95\% CIs. The Jadad scale was used to assess the quality of the RCTs [16], and studies with 3 or more points were identified as high quality [17]. The Newcastle-Ottawa Scale (NOS) was used to assess the quality of the observational studies [18], and studies with 7 or more points were deemed high quality. Any disagreements were resolved by consensus between the two reviewers (YW and FR).

\section{Statistical analysis}

This meta-analysis used the RR to estimate morbidity. All analyses used a random-effects model. The statistical values are reported with $95 \%$ CIs, and a twotailed $p<0.05$ was considered statistically significant. Heterogeneity among studies was tested by Cochran's $\mathrm{Q}$ test (reported with a $\chi^{2}$ value and $p$ value, with a $p<0.1$ considered statistically significant) and the $\mathrm{I}^{2}$ statistic $[19,20] . \mathrm{I}^{2}$ with values of 25,50 , and $75 \%$ demonstrated low, moderate, and high levels of heterogeneity, respectively [21]. We also conducted sensitivity analyses to assess the robustness of the results [22]. Subgroup analyses were conducted to explore sources of heterogeneity. Publication bias was assessed using Begg's and Egger's tests [23, 24]. All analyses were performed using Stata software version 12.0 (2011; Stata Corp., College Station, TX, USA).

\section{Results \\ Characteristics of selected studies}

A flow chart of the search strategy is illustrated in Fig. 1. The search retrieved 2,203 published studies. After removing duplicates, screening the title and abstract, and further evaluation, a total of 20 studies were included in our meta-analysis. Among these, one publication was excluded because it provided duplicated data [25]. The remaining 19 studies included two RCTs and 17 observational studies [12-15, 26-40]. Nine studies enrolled endometrial cancer patients, three enrolled ovarian cancer patients, and seven enrolled both. The cancer patients were from the population of women studied. The studies were carried out in eight countries, which included North America [12, 13, 26, 27, 29, 30, 32, 34, 39, 40], Europe $[14,15,28,31,33,35,36,38]$, and Asia [37]. Detailed information from the included studies is presented in Table 1 . 


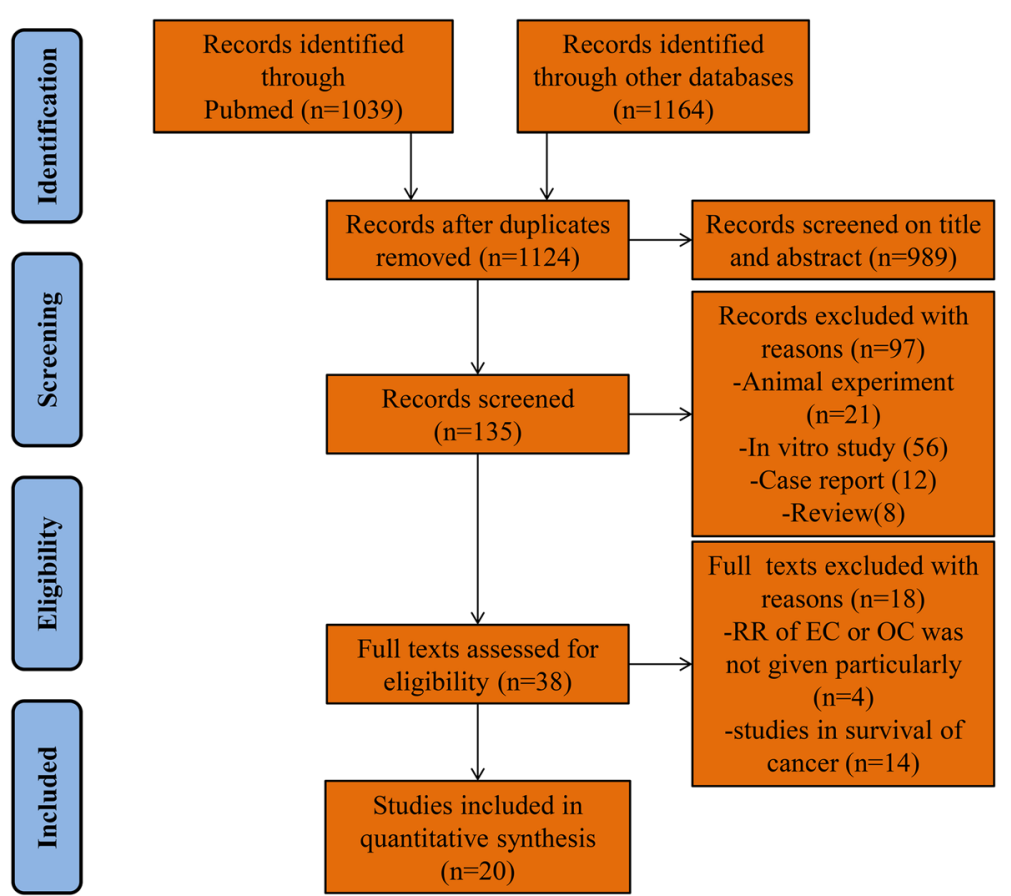

Fig. 1 Flow chart of our systematic review and meta-analysis

\section{Risk of ovarian cancer among statin users}

The meta-analysis included 10 studies of ovarian cancer. Our pooled analysis indicated that statin use did not reduce the risk of ovarian cancer $(R R=0.88,95 \%$ $\mathrm{CI}=0.76-1.03, p=0.12$ ) (Fig. 2). A moderately significant heterogeneity was observed among the studies $\left(x^{2}=20.00, \quad p=0.02, \quad I^{2}=55.0 \%\right)$. Sensitivity analyses were carried out by excluding studies one-by-one, and we found that the corresponding pooled RRs were not significantly altered, which affirmed the robustness of the result (Additional file 1: Figure S1). We conducted subgroup analyses of statin use and the risk of ovarian cancer based on study type, percentage of cancer cases, geographical study location (continent), and quality of studies. There was no significant association between statin use and the risk of ovarian cancer in the RCTs $(\mathrm{RR}=0.20,95 \% \mathrm{CI}=0.01-4.07$, $p=0.30)$, cohort studies $(\mathrm{RR}=0.98,95 \% \mathrm{CI}=0.77$ $1.24, p=0.85)$, or case-control studies $(\mathrm{RR}=0.82,95 \%$ $\mathrm{CI}=0.65-1.04, p=0.10)$. Subgroup analysis based on the percentage of cancer cases indicated that there was no significant association in groups with $\geq 1 \%$ $(\mathrm{RR}=0.76, \quad 95 \% \quad \mathrm{CI}=0.55-1.05, \quad p=0.10) \quad$ or $<1 \%$ $(\mathrm{RR}=0.97,95 \% \mathrm{CI}=0.83-1.14, p=0.74)$. Subgroup analysis of study location showed no reduced risk of ovarian cancer among statin users in North America $(\mathrm{RR}=0.88,95 \% \mathrm{CI}=0.69-1.12, p=0.29)$ or Europe $(\mathrm{RR}=0.90,95 \% \mathrm{CI}=0.72-1.12, p=0.34)$. Additionally, no decreased risk of ovarian cancer in statin users was observed, whether studies were high quality or low quality (Table 2). There was no evidence of publication bias (Begg's test, $p=0.86$, [Additional file 2: Figure S2A]; Egger's test, $p=0.34$, [Additional file 2: Figure S2B]).

\section{Risk of endometrial cancer among statin users}

The meta-analysis included 16 studies of endometrial cancer. There was no significant association between the use of statins and the risk of endometrial cancer $(\mathrm{RR}=0.88,95 \% \mathrm{CI}=0.78-1.00, p=0.05) \quad$ (Fig. 3). A moderately significant heterogeneity was observed among the studies $\left(x^{2}=47.50, P=0.00, \mathrm{I}^{2}=66.3 \%\right)$. Sensitivity analyses were carried out by excluding studies one-by-one, and we found that six studies [15, 26, 30, 32, 34, 35] seemed to affect the heterogeneity. Excluding any one of these studies from the overall analysis resulted in statistically significant results, but the upper limits of the $95 \%$ confidence interval were approximately 1.00 (Additional file 3: Figure S3). We also conducted subgroup analyses by study type, percentage of cancer cases, study location, and quality of studies. There was no significant association between statin use and the risk of endometrial cancer in the RCTs $(R R=0.72,95 \% C I=0.19-2.68, p=0.62)$, cohort studies ( $\mathrm{RR}=0.93,95 \% \mathrm{CI}=0.80-1.10, p=0.38)$, or case-control studies $(\mathrm{RR}=0.80,95 \% \mathrm{CI}=0.62-1.03$, $p=0.09$ ). Subgroup analysis based on the percentage of cancer cases indicated that there was no significant 


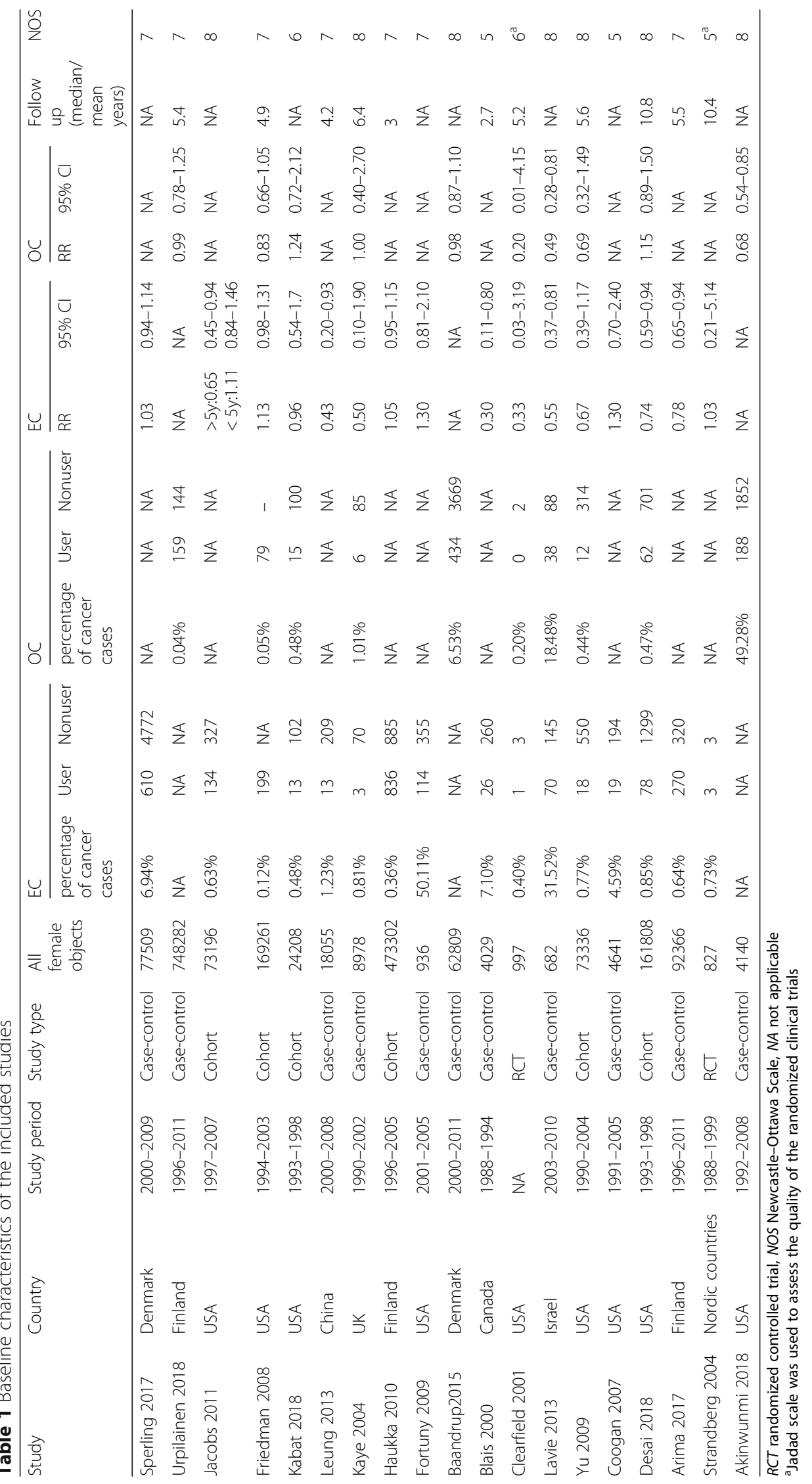




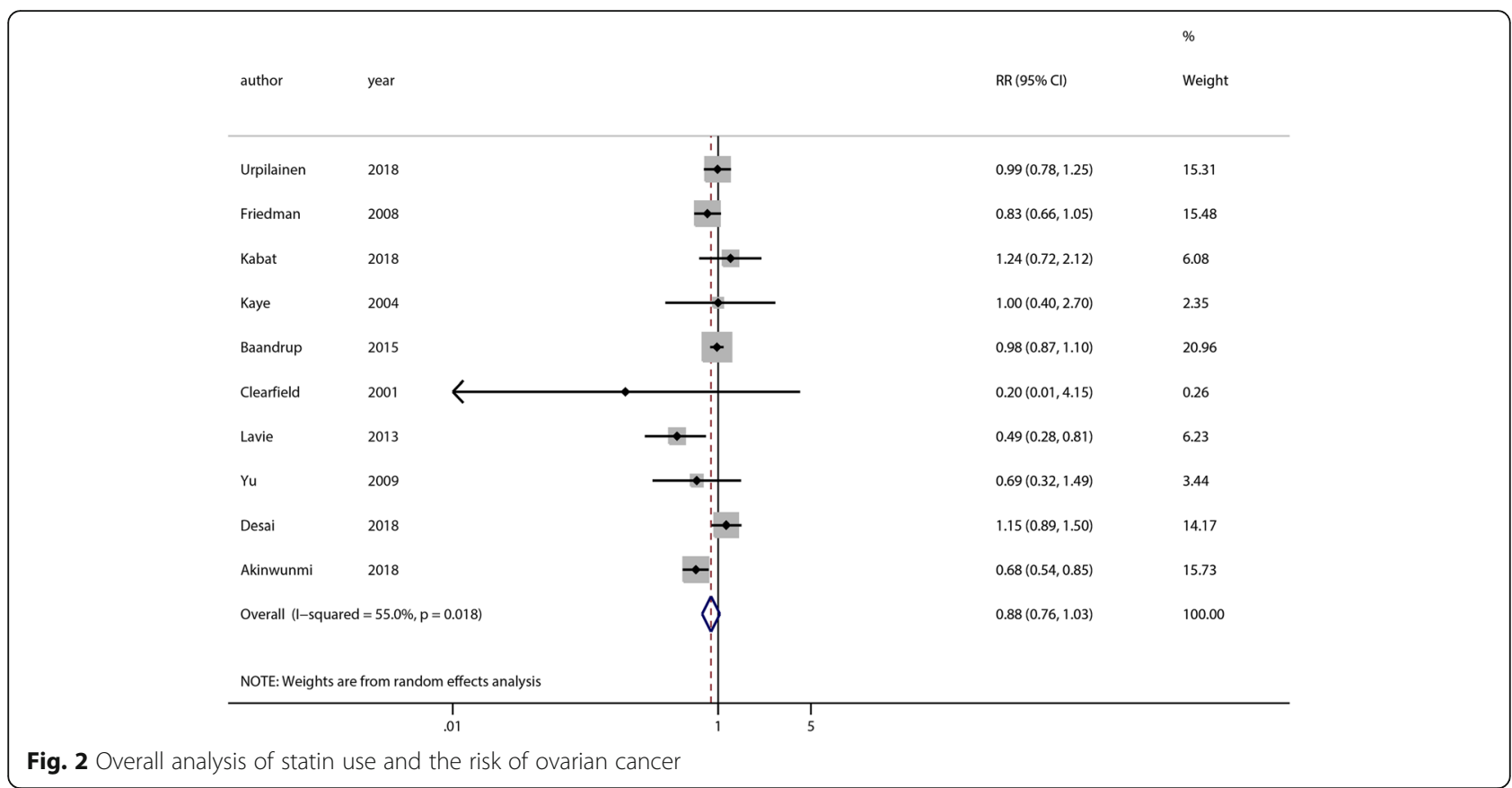

association in groups with $\geq 1 \%(\mathrm{RR}=0.79,95 \% \mathrm{CI}=$ $0.54-1.15, p=0.21) \quad$ or $<1 \% \quad(\mathrm{RR}=0.90,95 \% \quad \mathrm{CI}=$ $0.78-1.04, p=0.14$ ) of cancer cases in the study population. Subgroup analysis of studies based on geographical location showed a significantly reduced risk of endometrial cancer among statin users in Asia $(\mathrm{RR}=0.43,95 \% \mathrm{CI}=0.20-0.94, \quad p=0.03)$, but none in North America $(R R=0.90,95 \% \mathrm{CI}=0.73-$
$1.11, p=0.31)$ or Europe $(\mathrm{RR}=0.89,95 \% \mathrm{CI}=0.75-$ $1.05, p=0.18)$. Again, no significant association between statin use and risk of endometrial cancer was observed in studies of high quality or low quality (Table 2). There was evidence of potential publication bias (Begg's test, $P=0.434$, [Additional file 4: Figure S4A]; Egger's test, $p=0.03$, [Additional file 4: Figure $\mathrm{S} 4 \mathrm{~B}]$ ).

Table 2 Subgroup analyses of statin use and risk of endocrine-related gynecologic cancers

\begin{tabular}{|c|c|c|c|c|c|c|c|c|}
\hline \multirow[t]{2}{*}{ Characteristics } & \multicolumn{4}{|l|}{ Ovarian Cancer } & \multicolumn{4}{|c|}{ Endometrial Cancer } \\
\hline & Study number & $\mathrm{RR}(95 \% \mathrm{Cl})$ & $P$ value & Heterogeneity & Study number & $\mathrm{RR}(95 \% \mathrm{Cl})$ & $P$ value & Heterogeneity \\
\hline Study type & 10 & $0.88(0.76,1.03)$ & 0.12 & $55.0 \%$ & 16 & $0.88(0.78,1.00)$ & 0.05 & $66.3 \%$ \\
\hline $\mathrm{RCT}$ & 1 & $0.20(0.01,4.07)$ & 0.30 & $0.0 \%$ & 2 & $0.72(0.19,2.68)$ & 0.62 & $0.0 \%$ \\
\hline Cohort & 4 & $0.98(0.77,1.24)$ & 0.85 & $38.9 \%$ & 7 & $0.93(0.80,1.10)$ & 0.38 & $66.5 \%$ \\
\hline Case-control & 5 & $0.82(0.65,1.04)$ & 0.10 & $70.6 \%$ & 7 & $0.80(0.62,1.03)$ & 0.09 & $74.1 \%$ \\
\hline \multicolumn{9}{|c|}{ Percentage of cancer cases } \\
\hline$\geq 1 \%$ & 4 & $0.76(0.55,1.05)$ & 0.10 & $76.8 \%$ & 6 & $0.79(0.54,1.15)$ & 0.21 & $76.5 \%$ \\
\hline$<1 \%$ & 6 & $0.97(0.83,1.14)$ & 0.74 & $16.6 \%$ & 10 & $0.90(0.78,1.04)$ & 0.14 & $61.8 \%$ \\
\hline \multicolumn{9}{|l|}{ Study location } \\
\hline North America & 6 & $0.88(0.69,1.12)$ & 0.29 & $58.1 \%$ & 9 & $0.90(0.73,1.11)$ & 0.31 & $64.2 \%$ \\
\hline Europe & 4 & $0.90(0.72,1.12)$ & 0.34 & $52.7 \%$ & 6 & $0.89(0.75,1.05)$ & 0.18 & $72.2 \%$ \\
\hline Asia & 0 & - & - & - & 1 & $0.43(0.20,0.94)$ & 0.03 & $0.0 \%$ \\
\hline \multicolumn{9}{|l|}{ Quality of studies } \\
\hline High & 9 & $0.86(0.74,1.01)$ & 0.07 & $57.4 \%$ & 13 & $0.88(0.78,1.01)$ & 0.06 & $68.5 \%$ \\
\hline Low & 1 & $1.24(0.72,2.12)$ & 0.44 & $0.0 \%$ & 3 & $0.80(0.39,1.62)$ & 0.53 & $67.4 \%$ \\
\hline \multicolumn{9}{|c|}{ Dduration of statin use } \\
\hline long-term use & 5 & $0.73(0.51,1.04)$ & 0.08 & $58.9 \%$ & 6 & $0.79(0.58,1.08)$ & 0.14 & $58.2 \%$ \\
\hline Study in diabetics & 1 & $0.96(0.75,1.23)$ & 0.75 & $0.0 \%$ & 1 & $0.78(0.65,0.94)$ & 0.01 & $0.0 \%$ \\
\hline
\end{tabular}




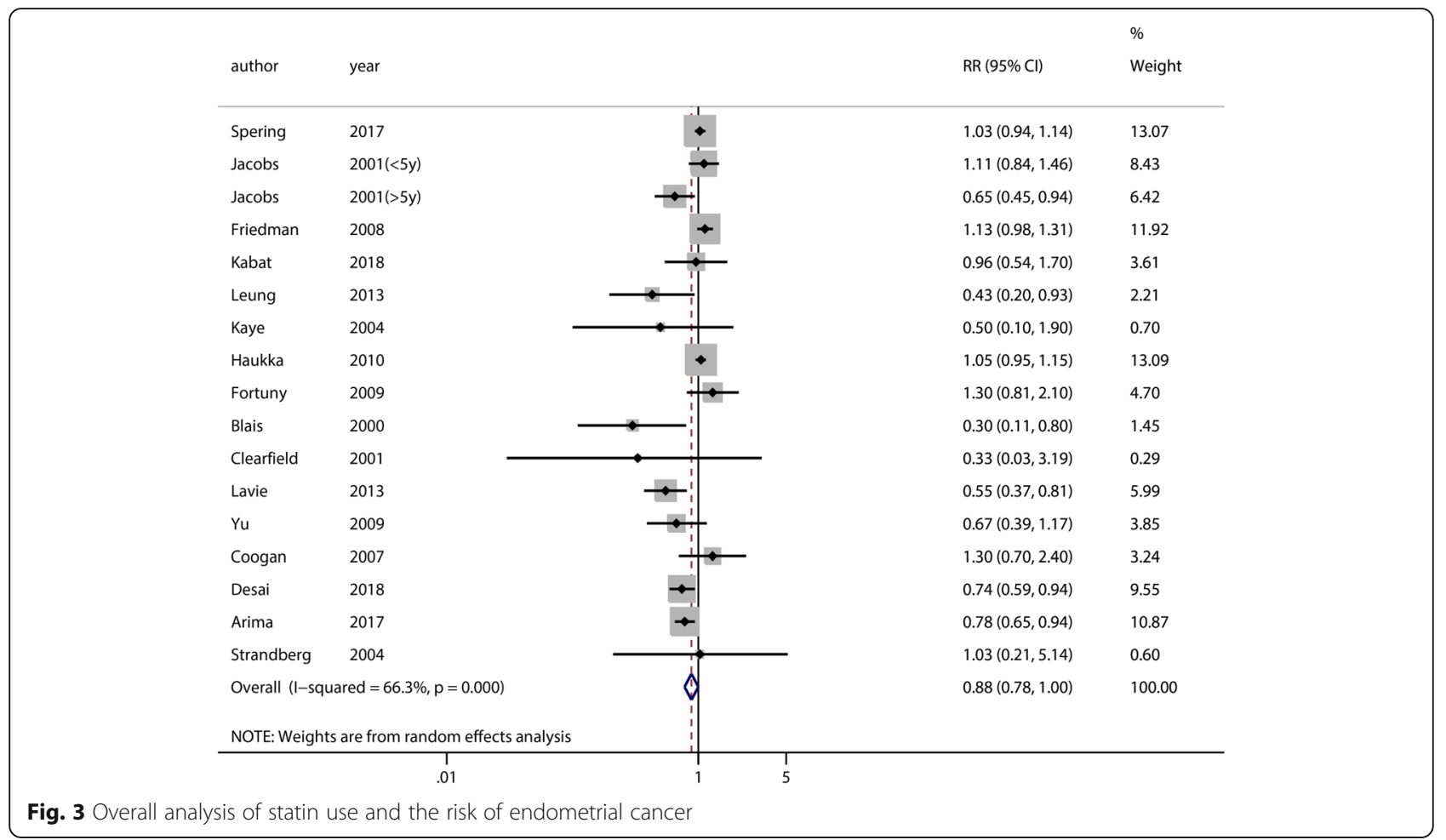

\section{Long-term statin use and the risk of ovarian or endometrial cancer}

The meta-analysis included five studies of ovarian cancer $[13,14,30,36,39]$ and six studies of endometrial cancer $[15,30,33,34,36,39]$ that analyzed long-term statin use ( $>5$ years). There was no association between long-term statin use ( $>5$ years) and the risk of endometrial cancer $(\mathrm{RR}=0.79,95 \% \mathrm{CI}=0.58-1.08, p=0.14)$ or ovarian cancer $(\mathrm{RR}=0.73,95 \% \mathrm{CI}=0.51-1.04, p=0.08)$ (Fig. 4).

\section{Discussion}

The association between statin use and the risk of endometrial or ovarian cancer remains controversial. Sperling et al. [15] reported no association between statin use and the risk of endometrial cancer, whereas Desai et al. [12] reported that statins reduced the risk of endometrial cancer. Some studies have reported a significant association between statin use and the risk of ovarian cancer $[13,25,36]$, but others found no such association [12, 31]. In 2014, Liu et al. [41] performed a meta-analysis of the effect of statin use on the risk of gynecological cancers and reported that statin use lowered the risk of ovarian cancer but not that of endometrial cancer. The debate has continued after that publication, and the results of more recent studies have also been inconsistent.

Our meta-analysis included 19 studies enrolling 1,999,362 women. Seven of these studies were published after the last meta-analysis in 2014 and included an additional 1,171,122 subjects. Our pooled analysis indicated that statin use did not reduce the risk of ovarian or endometrial cancer. In addition, we conducted subgroup analyses by study type, percentage of cancer cases, quality of studies, and study location to explore sources of heterogeneity. Two RCTs, six cohort studies and 11 case-control studies were included, and there was no significant association between the use of statins and the risk of ovarian or endometrial cancer based on study type. We used the Jadad scale and NOS criterion to assess the quality of the RCTs and observational studies, respectively. The two RCTs were deemed to be of high quality, with Jadad total scores $\geq 3$, and 14 observational studies had NOS scores $\geq 7$, identifying them as high quality. Subgroup analyses based on the quality of studies indicated that no significant association between statin use and the risk of endometrial cancer was observed in studies of high quality or low quality. The studies we included had different numbers of participants, most included large populations, and the percentage of cancer patients was low and varied. However, we found no significant differences in the association of statin use and the risk of ovarian or endometrial cancer in groups with percentages of cancer cases $\geq 1 \%$ versus groups with $<1 \%$. All of the above subgroup analyses supported our pooled conclusions. In the subgroup analyses based on study location, our results showed a similar tendency, with no reduced risk of ovarian or endometrial cancer in statin users, although a subgroup of 


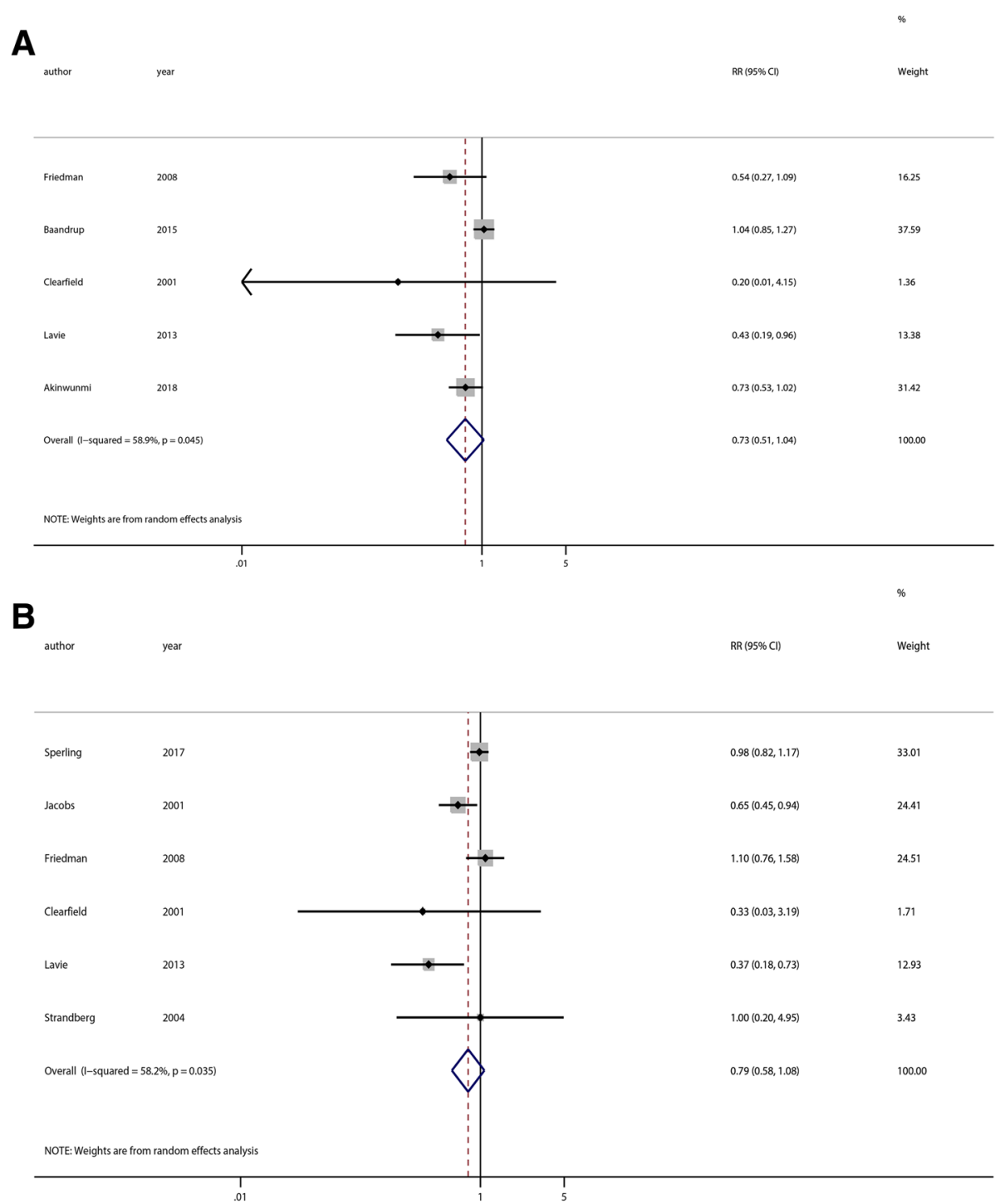

Fig. 4 Subgroup analyses of long-term statin use and the risk of ovarian and endometrial cancer. a Ovarian cancer; b Endometrial cancer

statin users in Asia did have a significantly reduced risk of endometrial cancer. This result might be due to regional disparities or racial differences. People in different countries have different dietary habits. Patel et al. reported that metabolic syndrome was less prevalent in Asian populations than it is in US populations [42]. Therefore, the effects of statin use may vary according to location. However, this meta-analysis subgroup (endometrial cancer patients in Asia) included only one study conducted in Asia, which enrolled fewer subjects than in other studies. Hence, the effects of statin use in endometrial cancer patients require further exploration, and our results require validation from additional RCTs enrolling large populations.

Ovarian and endometrial cancers may be associated with diabetes mellitus. Previous studies reported that patients with diabetes mellitus had an increased risk of ovarian cancer, and patients with ovarian cancer and diabetes had poor survival $[43,44]$. The incidence of endometrial cancer is increasing due to global increases in diabetes and obesity $[45,46]$, suggesting that we should target women with diabetes. We planned to conduct subgroup analyses of studies enrolling women with diabetes, but the number of studies was limited. Although the risk of endometrial cancer was significantly reduced in statin users, the results require validation with additional studies enrolling larger populations.

Studies investigating the effects of long-term statin use on ovarian or endometrial cancer have reported inconsistent results. Some studies found no association [34]. In contrast, the meta-analysis of Liu et al. [41] reported that long-term statin use significantly reduced the risk of ovarian cancer. Our subgroup analyses of long-term 
statin use included three more studies and 5,817 additional subjects than the previous meta-analysis [41] and showed no significant association between long-term statin use and the risk of ovarian or endometrial cancer. As we found no reduction in the risk of ovarian or endometrial cancer in statin users, long-term statin use also had no protective effect. The majority of studies of the effect of long-term statin use indicated no reduced risk of ovarian or endometrial cancer in statin users. Indeed, a recent observational study by Desai et al. [12] reported that the use of pravastatin was associated with an increased risk of ovarian cancer. This study enrolled a large number of female subjects $(161,808)$ and investigated the relationship between different drug types and cancer risk. Thus, the long-term effect of statins must still be considered unanswered, and more RCTs or highquality observational studies are needed to verify the effects of long-term statin use on ovarian or endometrial cancer.

Our study has some advantages. The meta-analysis included 19 studies enrolling 1,999,362 female subjects and 19,849 cancer cases. Our inclusion and exclusion criteria were based on PICOS criteria. The Jadad scale was used to assess the quality of the RCTs, and the NOS criterion was used to evaluate the quality of the observational studies; most of the studies were high quality. The 2014 meta-analysis [41] investigated the association between statin use and the risk of gynecological cancers. In contrast, we investigated the association between statin use and the risk of ovarian and endometrial cancers separately. Our meta-analysis included seven more studies and a larger number of participants than the previous metaanalysis, and our results differed from those of the earlier study. Our subgroup analyses based on study type, percentage of cancer cases, study location, and quality of studies largely supported our conclusions.

There were some limitations in our meta-analysis, which will be resolved in future studies. First, in the selected studies, women were prescribed statins primarily to treat metabolic syndrome, which may independently induce ovarian or endometrial cancer. As a result, it was not possible to pool metabolic status for the observational studies included in this meta-analysis. Moreover, in some studies, patients were prescribed other medications in addition to statins. As other medications may be associated with the risk of cancer, for example, the use of metformin or oral contraceptives may lower the risk of gynecologic cancer $[47,48]$, and hormone replacement therapy may influence the risk of gynecologic cancer [49], there was the possibility that our results had bias, which could be of concern. Hence, to confirm the conclusions of this meta-analysis, further RCTs enrolling larger populations to investigate the association between statin use and the risk of ovarian or endometrial cancer are needed.

Second, the heterogeneity of our study was significant. Our study included 2 RCTs and 17 observational studies; the two RCTs included 12 total cancer cases, whereas the largest studies included approximately 5,000 cancer cases [15]. Among the included observational studies, the study by Lavie et al. [36] is a case-control trial in which controls were individually matched to cases according to age, sex, clinic and ethnic group. Therefore, the study excluded many confounding factors, which might lead to a more reliable result. As it has been reported that statin use could reduce the risk of endometrial and ovarian cancers, we cannot ignore the important finding. Although our pooled analysis was negative, the majority of the studies we included were retrospective, which may contain selection, information, and confounding bias. More RCTs or high-quality observational studies are needed to confirm or update our meta-analysis. In addition, we extracted RR data directly or indirectly to represent the relationship between statin use and ovarian or endometrial cancer. However, each of the included studies utilized different study designs according to the different statins or different statin doses. Most studies observed the outcomes of overall statin use (rather than that of a particular drug) and cancer risk. Other studies classified statins as lipophilic or hydrophilic $[13,37]$ or separately observed different drugs $[15,33,35,39]$. As different statins have different half-lives, different types or doses of statin have been proposed to contribute differently to clinical outcomes. However, the results could not be combined because of such differences in the included studies. Most studies did not record the duration of statin use, and other studies evaluating statin duration were not coincident in the observation time. Thus, we could not combine the results according to the duration of statin use specifically, but only extracted data for long-term statin use ( $>5$ years). Moreover, the studies we included differed in terms of research methods, follow-up time, and source of the population; any of these factors may have induced study heterogeneity.

Third, the sensitivity analysis indicated that excluding studies one-by-one did not alter the corresponding pooled RRs in the ovarian cancer group, which affirmed the robustness of the result. However, for the studies of endometrial cancer, we found that six studies seemed to affect the heterogeneity. When we excluded any one of these six studies from the overall analysis, statistically significant results were obtained. However, the upper limits of the $95 \%$ confidence intervals were approximately 1.00. Thus, as we did not think that excluding so many studies was appropriate, we maintained the original pooled results. In addition, when we conducted 
analyses of statin use and the risk of ovarian cancer, there was no evidence of publication bias. However, in the overall analyses of endometrial cancer, although there was no significance in Begg's test, Egger's test was statistically significant, which indicated a potential publication bias. The most likely explanation is the preferential publication of positive findings.

Fourth, the number of RCTs investigating statin use and the risk of ovarian or endometrial cancer was limited. The search retrieved two RCTs with 12 cancer cases but 17 observational studies with 19,837 cancer cases. Moreover, in these RCTs, statins were used to treat patients with coronary heart disease or hyperlipemia. Cancer was not a primary outcome. Hence, the results from the two RCTs were not powerful enough to conclude the outcome of cancers. Thus, further RCTs investigating statin use and the risk of ovarian or endometrial cancer will be required to confirm these results.

\section{Conclusions}

This meta-analysis identified no reduction in the risk of ovarian or endometrial cancer for patients who used statins. In addition, the long-term use of statins ( $>5$ years) was not associated with a reduction in the risk of ovarian or endometrial cancer. To confirm the conclusions of this meta-analysis, further RCTs enrolling larger populations to investigate the association between statin use and the risk of ovarian or endometrial cancer are needed.

\section{Additional files}

Additional file 1: Figure S1. Sensitivity analyses based on statin use and the risk of ovarian cancer. (TIF $1190 \mathrm{~kb}$ )

Additional file 2: Figure S2. Publication bias in terms of statin use and the risk of ovarian cancer. (A) Begg's test; (B) Egger's test. (TIF 1616 kb)

Additional file 3: Figure S3. Sensitivity analyses based on statin use and the risk of endometrial cancer. (TIF $1344 \mathrm{~kb}$ )

Additional file 4: Figure S4. Publication bias in terms of statin use and the risk of endometrial cancer. (A) Begg's test; (B) Egger's test. (TIF $1658 \mathrm{~kb})$

\section{Abbreviations}

Cl: Confidence interval; HMG CoA: 3-hydroxy-3-methylglutaryl coenzyme A; HR: Hazard ratio; NA: Not applicable; NOS: Newcastle-Ottawa Scale; OR: Odds ratio; PICOS: Population, intervention, comparison, outcomes, and study design; PRISMA: Preferred Reporting Items for Systematic Reviews and Meta -Analyses; RCT: Randomized control trial; RR: Relative risk;

Statins: Hydroxymethylglutaryl-CoA reductase inhibitors

\section{Acknowledgements}

Not applicable.

\section{Authors' contributions}

LO and YW conceived the study idea and devised the study methodology. FR and ZS participated in the design and coordination of the study. YW and FR screened identified literature, conducted data extraction, analysed the narrative review findings and conducted the meta-analysis. PC and SL provided specific support in quantitative data analysis. YW wrote the manuscript. All authors contributed to improving the manuscript, read and approved the version of the manuscript to be published. All authors take responsibility for appropriate content.

\section{Funding}

The project was supported by National Natural Science Foundation of China (Grant number 81501235). The funding body had no role in the design of the study, collection, analysis, or interpretation of the data, or in the writing of the manuscript.

\section{Availability of data and materials}

Input data for the analyses are available from the corresponding author on request.

Ethics approval and consent to participate

Not applicable.

\section{Consent for publication}

Not applicable.

\section{Competing interests}

The authors declare that they have no competing interests.

Received: 24 October 2018 Accepted: 18 July 2019

Published online: 24 July 2019

References

1. Siegel RL, Miller KD, Jemal A. Cancer statistics, 2017. CA Cancer J Clin. 2017; 67(1):7-30.

2. Arend RC, Londono-Joshi Al, Straughn JM Jr, Buchsbaum DJ. The Wht/ beta-catenin pathway in ovarian cancer: a review. Gynecol Oncol. 2013; 131(3):772-9.

3. Sorosky Jl. Endometrial cancer. Obstet Gynecol. 2012;120(2 Pt 1):383-97.

4. Esposito K, Chiodini P, Colao A, Lenzi A, Giugliano D. Metabolic syndrome and risk of cancer: a systematic review and meta-analysis. Diabetes Care. 2012;35(11):2402-11

5. lannelli F, Lombardi R, Milone MR, Pucci B, De Rienzo S, Budillon A, Bruzzese F. Targeting mevalonate pathway in Cancer treatment: repurposing of statins. Recent Pat Anticancer Drug Discov. 2018;13(2):184-200.

6. Kobayashi Y, Kashima H, Rahmanto YS, Banno K, Yu Y, Matoba Y, Watanabe K, lijima M, Takeda T, Kunitomi H, et al. Drug repositioning of mevalonate pathway inhibitors as antitumor agents for ovarian cancer. Oncotarget. 2017;8(42):72147-56

7. Zeybek B, Costantine M, Kilic GS, Borahay MA. Therapeutic roles of statins in gynecology and obstetrics: the current evidence. Reprod Sci. 2018;25(6):802-17.

8. Schointuch MN, Gilliam TP, Stine JE, Han X, Zhou C, Gehrig PA, Kim K, Bae -Jump VL. Simvastatin, an HMG-CoA reductase inhibitor, exhibits anti -metastatic and anti-tumorigenic effects in endometrial cancer. Gynecol Oncol. 2014:134(2):346-55.

9. Kato S, Liberona MF, Cerda-Infante J, Sanchez M, Henriquez JF, Bizama C, Bravo ML, Gonzalez P, Gejman R, Branes J, et al. Simvastatin interferes with cancer 'stem-cell' plasticity reducing metastasis in ovarian cancer. Endocr Relat Cancer. 2018. https://doi.org/10.1530/erc-18-0132.

10. Laezza C, Malfitano AM, Proto MC, Esposito I, Gazzerro P, Formisano P, Pisanti S, Santoro A, Caruso MG, Bifulco M. Inhibition of 3-hydroxy-3 -methylglutaryl-coenzyme a reductase activity and of Ras farnesylation mediate antitumor effects of anandamide in human breast cancer cells. Endocr Relat Cancer. 2010:17(2):495-503.

11. Cuello FM, Kato CS, Diaz SD, Owen G. Effects of statins in cancer. Rev Med Chil. 2013;141(2):227-36.

12. Desai P, Wallace R, Anderson ML, Howard BV, Ray RM, Wu C, Safford M, Martin LW, Rohan T, Manson JE, et al. An analysis of the association between statin use and risk of endometrial and ovarian cancers in the Women's Health Initiative. Gynecol Oncol. 2018;148(3):540-6.

13. Akinwunmi B, Vitonis AF, Titus L, Terry KL, Cramer DW. Statin therapy and association with ovarian Cancer risk in the New England case control (NEC) study. Int J Cancer. 2018. https://doi.org/10.1002/ijc.31758.

14. Baandrup L, Dehlendorff C, Friis S, Olsen JH, Kjaer SK. Statin use and risk for ovarian cancer: a Danish nationwide case-control study. Br J Cancer. 2015. 112(1):157-61 
15. Sperling CD, Verdoodt F, Friis S, Dehlendorff C, Kjaer SK. Statin use and risk of endometrial cancer: a nationwide registry-based case-control study. Biomed Res Int. 2017;96(2):144-9.

16. Jadad AR, Moore RA, Carroll D, Jenkinson C, Reynolds DJ, Gavaghan DJ, McQuay HJ. Assessing the quality of reports of randomized clinical trials: is blinding necessary? Control Clin Trials. 1996;17(1):1-12.

17. Clark HD, Wells GA, Huet C, McAlister FA, Salmi LR, Fergusson D, Laupacis A. Assessing the quality of randomized trials: reliability of the Jadad scale. Control Clin Trials. 1999;20(5):448-52.

18. Stang A. Critical evaluation of the Newcastle-Ottawa scale for the assessment of the quality of nonrandomized studies in meta-analyses. Eur J Epidemiol. 2010;25(9):603-5.

19. Laird NM, Mosteller F. Some statistical methods for combining experimental results. Int J Technol Assess Health Care. 1990;6(1):5-30.

20. Dickersin K, Berlin JA. Meta-analysis: state-of-the-science. Epidemiol Rev. 1992;14:154-76.

21. Higgins JP, Thompson SG, Deeks JJ, Altman DG. Measuring inconsistency in meta-analyses. Bmj. 2003;327(7414):557-60.

22. Copas J, Shi JQ. Meta-analysis, funnel plots and sensitivity analysis. Biostatistics. 2000;1(3):247-62.

23. Egger M, Davey Smith G, Schneider M, Minder C. Bias in meta-analysis detected by a simple, graphical test. Bmj. 1997;315(7109):629-34.

24. Begg CB, Mazumdar M. Operating characteristics of a rank correlation test for publication bias. Biometrics. 1994;50(4):1088-101.

25. Rennert G, Rennert HS, Pinchev M, Lavie O. The effect of bisphosphonates on the risk of endometrial and ovarian malignancies. Gynecol Oncol. 2014;133(2):309-13.

26. Coogan PF, Rosenberg L, Strom BL. Statin use and the risk of 10 cancers. Epidemiology. 2007;18(2):213-9.

27. Yu O, Boudreau DM, Buist DS, Miglioretti DL. Statin use and female reproductive organ cancer risk in a large population-based setting. Cancer Causes Control. 2009;20(5):609-16.

28. Kaye JA, Jick H. Statin use and cancer risk in the general practice research database. Br J Cancer. 2004:90(3):635-7.

29. Kabat GC, Kim MY, Chlebowski RT, Vitolins MZ, Wassertheil-Smoller S, Rohan TE. Serum lipids and risk of obesity-related cancers in postmenopausal women. Cancer Causes Control. 2018;29(1):13-24.

30. Friedman GD, Flick ED, Udaltsova N, Chan J, Quesenberry CP Jr, Habel LA. Screening statins for possible carcinogenic risk: up to 9 years of follow-up of 361,859 recipients. Pharmacoepidemiol Drug Saf. 2008;17(1):27-36.

31. Urpilainen E, Marttila M, Hautakoski A, Arffman M, Sund R, llanne-Parikka P, Arima R, Kangaskokko J, Puistola U, Laara E, et al. The role of metformin and statins in the incidence of epithelial ovarian cancer in type 2 diabetes: a cohort and nested case-control study. Bjog. 2018;125(8):1001-8.

32. Fortuny J, Sima C, Bayuga S, Wilcox H, Pulick K, Faulkner S, Zauber AG, Olson $\mathrm{SH}$. Risk of endometrial cancer in relation to medical conditions and medication use. Cancer Epidemiol Biomark Prev. 2009;18(5):1448-56.

33. Strandberg TE, Pyorala K, Cook TJ, Wilhelmsen L, Faergeman $\mathrm{O}$, Thorgeirsson G, Pedersen TR, Kjekshus J. Mortality and incidence of cancer during 10-year follow-up of the Scandinavian simvastatin survival study (4S). Lancet. 2004;364(9436):771-7.

34. Jacobs EJ, Newton CC, Thun MJ, Gapstur SM. Long-term use of cholestero -lowering drugs and cancer incidence in a large United States cohort. Cancer Res. 2011;71(5):1763-71.

35. Haukka J, Sankila R, Klaukka T, Lonnqvist J, Niskanen L, Tanskanen A Wahlbeck K, Tiihonen J. Incidence of cancer and statin usage--record linkage study. Int J Cancer. 2010;126(1):279-84.

36. Lavie O, Pinchev M, Rennert HS, Segev Y, Rennert G. The effect of statins on risk and survival of gynecological malignancies. Gynecol Oncol. 2013;130(3):615-9.

37. Leung HW, Chan AL, Lo D, Leung JH, Chen HL. Common cancer risk and statins: a population-based case-control study in a Chinese population. Expert Opin Drug Saf. 2013;12(1):19-27.

38. Arima R, Marttila M, Hautakoski A, Arffman M, Sund R, llanne-Parikka P, Kangaskokko J, Laara E, Puistola U, Hinkula M. Antidiabetic medication, statins and the risk of endometrioid endometrial cancer in patients with type 2 diabetes. Gynecol Oncol. 2017;146(3):636-41.

39. Clearfield M, Downs JR, Weis S, Whitney EJ, Kruyer W, Shapiro DR, Stein EA Langendorfer A, Beere PA, Gotto AM. Air force/Texas coronary atherosclerosis prevention study (AFCAPS/TexCAPS): efficacy and tolerability of long-term treatment with lovastatin in women. J Womens Health Gend Based Med. 2001;10(10):971-81.
40. Blais L, Desgagne A, LeLorier J. 3-Hydroxy-3-methylglutaryl coenzyme a reductase inhibitors and the risk of cancer: a nested case-control study. Arch Intern Med. 2000;160(15):2363-8.

41. Liu Y, Qin A, Li T, Qin X, Li S. Effect of statin on risk of gynecologic cancers: a meta-analysis of observational studies and randomized controlled trials. Gynecol Oncol. 2014;133(3):647-55.

42. Patel A, Huang KC, Janus ED, Gill T, Neal B, Suriyawongpaisal P, Wong E, Woodward M, Stolk RP. Is a single definition of the metabolic syndrome appropriate?--a comparative study of the USA and Asia. Atherosclerosis. 2006;184(1):225-32.

43. Lee JY, Jeon I, Kim JW, Song YS, Yoon JM, Park SM. Diabetes mellitus and ovarian cancer risk: a systematic review and meta-analysis of observational studies. Int J Gynecol Cancer. 2013;23(3):402-12.

44. Shah MM, Erickson BK, Matin T, McGwin G Jr, Martin JY, Daily LB, Pasko D, Haygood CW, Fauci JM, Leath CA 3rd. Diabetes mellitus and ovarian cancer: more complex than just increasing risk. Gynecol Oncol. 2014;135(2):273-7.

45. Jenabi E, Poorolajal J. The effect of body mass index on endometrial cancer: a meta-analysis. Public Health. 2015;129(7):872-80.

46. Pearson-Stuttard J, Zhou B, Kontis V, Bentham J, Gunter MJ, Ezzati M. Worldwide burden of cancer attributable to diabetes and high body -mass index: a comparative risk assessment. Lancet Diabetes Endocrinol. 2018;6(6):e6-e15.

47. Wen Q, Zhao Z, Wen J, Zhou J, Wu J, Lei S, Miao Y. The association between metformin therapy and risk of gynecological cancer in patients: two meta-analyses. Eur J Obstet Gynecol Reprod Biol. 2019;237:33-41.

48. Cho MK. Use of combined Oral contraceptives in Perimenopausal women. Chonnam Med J. 2018;54(3):153-8.

49. Sjogren LL, Morch LS, Lokkegaard E. Hormone replacement therapy and the risk of endometrial cancer: a systematic review. Maturitas. 2016;91:25-35.

\section{Publisher's Note}

Springer Nature remains neutral with regard to jurisdictional claims in published maps and institutional affiliations.
Ready to submit your research? Choose BMC and benefit from:

- fast, convenient online submission

- thorough peer review by experienced researchers in your field

- rapid publication on acceptance

- support for research data, including large and complex data types

- gold Open Access which fosters wider collaboration and increased citations

- maximum visibility for your research: over $100 \mathrm{M}$ website views per year

At $\mathrm{BMC}$, research is always in progress.

Learn more biomedcentral.com/submissions 